\title{
Crystal structure of the elF4A-PDCD4 complex
}

\author{
Jeong Ho Changa,b, Yong Hyun Cho a,b, Sun Young Sohnn, \\ Sung Key Jang ${ }^{b}$, and Yunje Cho ${ }^{\mathrm{a}, \mathrm{b}, 1}$
} aNational Creative Initiatives for Structural Biology and bDepartment of Life Science, Pohang University of Science and Technology, Hyo-ja dong, San31,
Pohang, KyungBook, South Korea; and 'Biosciences Division, Structural Biology Center, Argonne National Laboratory, 9700 South Cass Avenue, Argonne, IL 60439

Edited by Nancy H. Colburn, National Cancer Institute-Frederick Cancer Research and Development Center, Frederick, MD, and accepted by the Editorial Board January 7, 2009 (received for review August 21, 2008)

Tumor suppressor programmed cell death protein 4 (PDCD4) inhibits the translation initiation factor elF4A, an RNA helicase that catalyzes the unwinding of secondary structure at the $5^{\prime}$-untranslated region of mRNAs and controls the initiation of translation. Here, we determined the crystal structure of the human elF4A and PDCD4 complex. The structure reveals that one molecule of PDCD4 binds to the two elF4A molecules through the two different binding modes. While the two MA3 domains of PDCD4 bind to one elF4A molecule, the C-terminal MA3 domain alone of the same PDCD4 also interacts with another elF4A molecule. The elF4A-PDCD4 complex structure suggests that the MA3 domain(s) of PDCD4 binds perpendicular to the interface of the two domains of elF4A, preventing the domain closure of elF4A and blocking the binding of RNA to elF4A, both of which are required events in the function of elF4A helicase. The structure, together with biochemical analyses, reveals insights into the inhibition mechanism of elF4A by PDCD4 and provides a framework for designing chemicals that target elF4A.

translation inhibition | tumor suppressor | RNA helicase |

domain closure | MA3 domain

P rogrammed cell death protein 4 (PDCD4) is a translation inhibitor that suppresses neoplastic transformation in cultured cells and transgenic mice (1-3). Loss or reduced expression of PDCD4 has been implicated in the development and progression of a variety of aggressive human cancers (4-6). PDCD4 is regulated by the $\mathrm{S} 6 \mathrm{~K} 1$ kinase and the $\mathrm{SCF}^{\beta \mathrm{TRCP}}$ ubiquitin ligase in response to the activation of the mTOR pathway by mitogens, and the controlled degradation of PDCD4 is essential for efficient protein synthesis and consequently for cell growth and proliferation (7).

PDCD4 is believed to perform its tumor suppressor function primarily through interaction with eIF4A and eIF4G, which are components of mRNA-binding complex eIF4F (8). eIF4A is a DEAD-box RNA helicase, with two domains, that unwinds the secondary structures in $5^{\prime}$-untranslated region (UTR) and cap of mRNA and thereby facilitates ribosome scanning (8). eIF4G is an adaptor protein that coordinates assembly of translation factors and the small ribosomal subunit (8). PDCD4 is believed to inhibit cap-dependent translation by directly inhibiting the helicase activity of eIF4A or by competing with eIF4G for binding to eIF4A and preventing assembly into a eukaryotic initiation complex, eIF4F, or both $(1,9,10)$.

PDCD4 is formed with the two MA3 domains at its middle (mMA3) and C-terminal regions (cMA3) (9, 11-13). Mutational and NMR binding analysis have shown that PDCD4 uses both MA3 domains to interact with eIF4A and prevents translation (9, 10). However, other studies have demonstrated that the cMA3 domain alone is sufficient for the inhibition of RNA helicase and translation (12). The interactions between eIF4A and PDCD4 have been analyzed in several mutational and NMR mapping studies $(1,9,10)$. Nevertheless, it is unclear from these studies how PDCD4 inhibits eIF4A at the molecular level. To elucidate the mechanism by which PDCD4 recognizes eIF4A and inhibits its helicase activity and translation, we determine the crystal structure of the human eIF4A-PDCD4 complex. The structure shows that PDCD4 inhibits the eIF4A through the direct binding in two different binding modes. In both binding modes, one or two MA3 domains bind to interdomain cleft and lock the domain closure of eIF4A while masking the RNA-binding surface of eIF4A. Using biochemical analyses, we demonstrate the importance of the two different binding modes of PDCD4 on eIF4A and provide insights into PDCD4-mediated helicase inhibition and translation inhibition.

\section{Results and Discussion}

Human elF4A and PDCD4 Form a 2:1 Complex. Our initial attempt to crystallize the full-length human eIF4A and PDCD4 was not successful. To isolate a protein complex suitable for crystallization, we construct a truncated form of human eIF4A (residues 20-406, eIF4A $\Delta \mathrm{N}$ ) and PDCD4 (residues 164-469, PDCD4 $\Delta \mathrm{N}$ ) in which the $\mathrm{N}$-terminal region was removed from both proteins [supporting information (SI) Fig. S1 $A$ ]. The eIF4A $\Delta \mathrm{N}$ exhibits slightly better RNA helicase activity compared with that of full-length eIF4A, and PDCD $4 \Delta \mathrm{N}$ also efficiently inhibits the helicase activity of eIF4A and in vitro translation (Fig. S1 $B$ and $C)$. Assuming that eIF4A $\Delta \mathrm{N}$ and PDCD $4 \Delta \mathrm{N}$ would interact with a 1:1 stoichiometry, we predicted the molecular mass of the eIF4A $\Delta \mathrm{N}-\mathrm{PDCD} 4 \Delta \mathrm{N}$ complex to be $78 \mathrm{kDa}$. However, gel filtration analysis repeatedly revealed that the apparent molecular mass of the complex is $130 \mathrm{kDa}$ (Fig. S1D). Additional analyses using analytical ultracentrifugation and isothermal titration calorimetry (ITC) further support that the two proteins form a 2:1 complex (Figs. S1E and $\mathrm{S} 2$ and Table S1). We determined the structure of this complex by single-wavelength anomalous dispersion at $2.8-\AA$ resolution (Table S2).

Overall Structure of the elF4A-PDCD4 Complex. The structure shows an unexpected 2:1 stoichiometry between eIF4A $\Delta \mathrm{N}$ and PDCD $4 \Delta \mathrm{N}$, which accounts for the observed mass of $130 \mathrm{kDa}$ (Fig. 1A). Because there are two independent complexes in the crystallographic asymmetric unit and each complex contains one molecule of PDCD $4 \Delta \mathrm{N}$ bound to the two eIF $4 \mathrm{~A} \Delta \mathrm{N}$, it is unlikely that the 2:1 complex formation between eIF4A and PDCD4 in a crystal is caused by the packing effect (Fig. S3). The two PDCD4 molecules in an asymmetric unit exhibit $0.4-A$ root mean square deviation (rmsd) values for all modeled $\mathrm{C}^{\alpha}$ atoms, and the first and second eIF4A proteins in a complex show $0.6-\AA$ and $0.7-\AA$ rmsd values compared with the equivalent eIF4A molecules in another complex, respectively. Despite high similarities

Author contributions: J.H.C., Y.H.C., and Y.C. designed research; J.H.C., Y.H.C., S.Y.S., J.M.C. A.K., Y.C.K., and Y.C. performed research; J.H.C., Y.H.C., S.Y.S., S.K.J., and Y.C. analyzed data; and Y.C. wrote the paper.

The authors declare no conflict of interest.

This article is a PNAS Direct Submission. N.H.C. is a guest editor invited by the Editorial Board.

Data deposition: The atomic coordinates have been deposited in the Protein Data Bank www.pdb.org (PDB ID code 2ZU6).

${ }^{1}$ To whom correspondence should be addressed. E-mail: yunje@postech.ac.kr.

This article contains supporting information online at www.pnas.org/cgi/content/full/ 0808275106/DCSupplemental. 
A

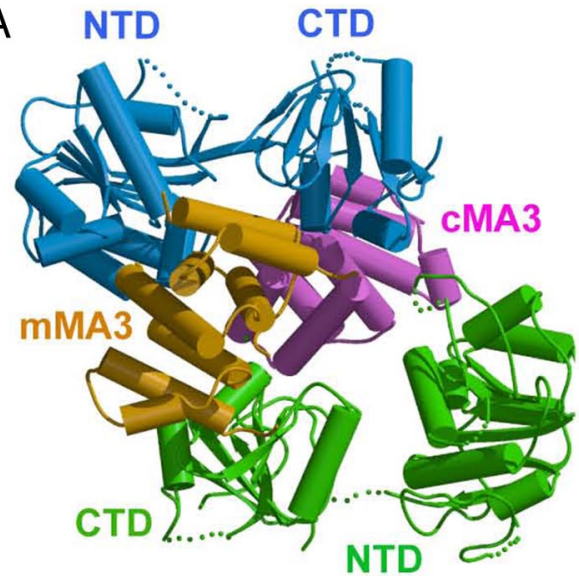

B

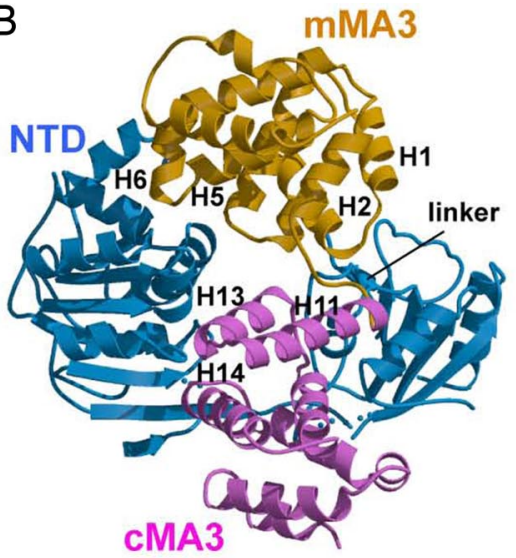

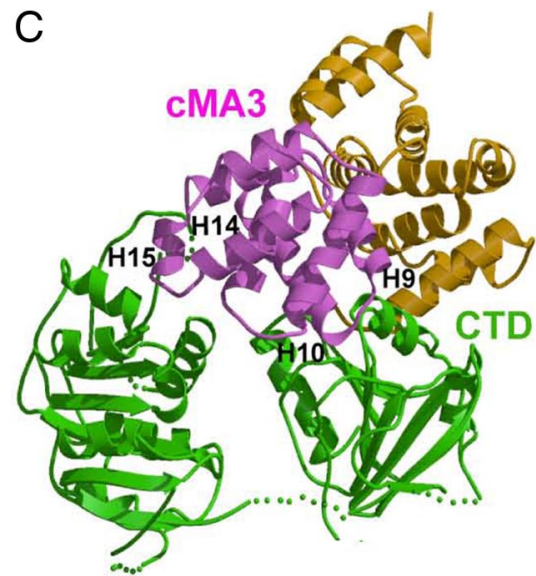

D

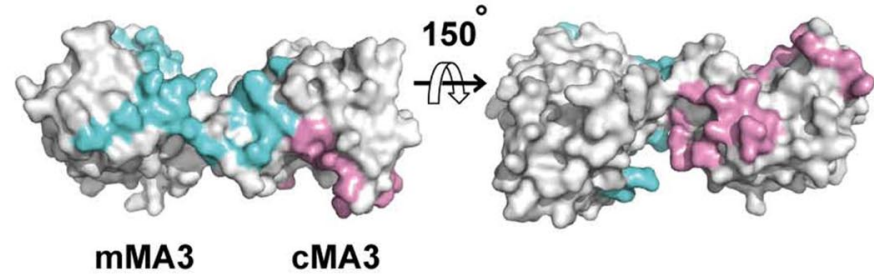

$E$

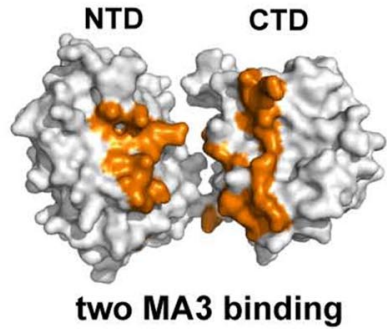

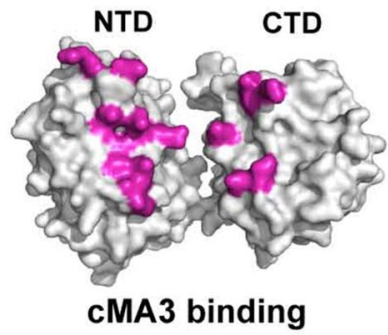

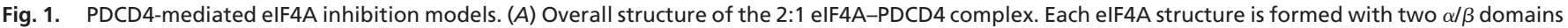

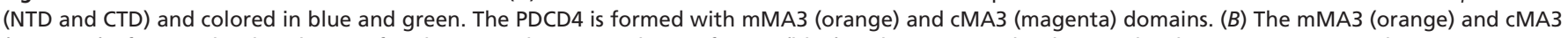

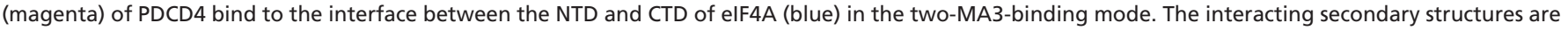

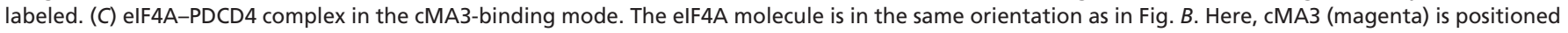

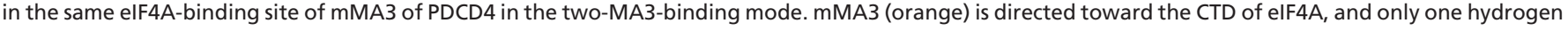

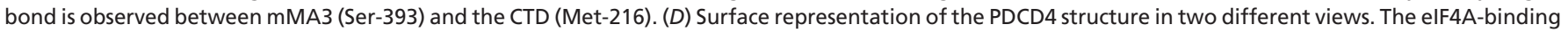

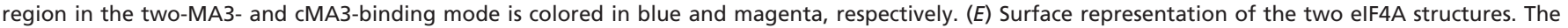
PDCD4-binding site in the two-MA3-binding mode is colored orange (Left) and in the cMA3-binding mode is colored magenta (Right).

between each molecule, the two complexes show relatively high rmsd values $(1.3 \AA)$ for all $\mathrm{C}^{\alpha}$ atoms because the relative orientation of one eIF4A (eIF4A-2) toward PDCD4 in a complex is slightly different from that of the equivalent eIF4A (eIF4A-4) in another complex (Fig. S3B).

While both the mMA3 domain and cMA3 domain of PDCD $4 \Delta \mathrm{N}$ interact with one eIF $4 \mathrm{~A} \Delta \mathrm{N}$ molecule, the same cMA3 domain also binds to another eIF4A $\Delta \mathrm{N}$ molecule (Fig. $1 A)$. As a result, the cMA3 domain uses a different surface to interact with the two eIF4A $\Delta \mathrm{N}$ molecules (Fig. 1D). However, the same surface of the two eIF4A molecules is targeted in both binding modes using the two MA3 domains or the cMA3 domain only (Fig. $1 E$ ). The human eIF4A structure consists of the two RecA-like $\alpha / \beta$ domains joined by a nine-residue interdomain linker. Despite their overall similarities, the two eIF4A structures in a complex exhibit slight differences in terms of the relative orientation of the two domains (Fig. S3C).

The PDCD4 forms a dumbbell shape in which each MA3 domain is connected through a 15-residue linker. The mMA3 and cMA3 domains of PDCD4, which are formed with eight and nine $\alpha$-helices, respectively, are asymmetrically arranged in PDCD4, and no interactions are observed between the two domains (Fig. S4A). The mMA3 domain can be transformed to cMA3 by a $74^{\circ}$ rotation and a $7.7-\AA$ translation (Fig. $\mathrm{S} 4 B$ ). The mMA3 and cMA3 domains of PDCD4 run perpendicular to the interface of the two domains of eIF4A and bind shallowly to the interface between the N-terminal domain (NTD) and Cterminal domain (CTD) of eIF4A (Fig. $1 A$ and $B$ ). Because of the PDCD4 binding, the two domains of eIF4A are separated, and no interactions are observed between the two domains (Fig.
$1 A-C)$. The relative orientation of the NTD and CTD of human eIF4A bound to PDCD4 is significantly different from that of yeast apoeIF4A and the eIF4G-bound eIF4A structures: the two domains of human eIF $4 \mathrm{~A} \Delta \mathrm{N}$ bound to PDCD $4 \Delta \mathrm{N}$ form a more closed conformation compared with those of yeast apoeIF4A and the eIF4G-bound eIF4A $(14,15)$ (Fig. S5).

Both mMA3 and CMA3 Domains of PDCD4 Bind to elF4A. Because of the 1:2 stoichiometry between PDCD $4 \Delta \mathrm{N}$ and eIF4A $\Delta \mathrm{N}$, we observed two different types of binding modes: the two-MA3binding mode, in which both mMA3 and cMA3 domains bind to one eIF4A molecule, and the cMA3-binding mode, in which only the cMA3 domain is involved in binding to another eIF4A molecule (Figs. $1 B$ and $C$ and 2). In the two-MA3-binding mode, the two MA3 domains interacted with both the NTD and CTD of eIF4A, with the buried surface area of 3,567 $\AA^{2}$ in three interfaces, mMA3-NTD, mMA3-CTD, and cMA3-CTD (Fig. 2 $A, C$, and $E$ ). The mMA3 binds to the NTD and CTD of eIF4A primarily through a combination of ion pairs and hydrogen bonds. In the mMA3-NTD interface, the H3-H4 and H5-H6 loops of mMA3 fit into the shallow grooves formed by the $\mathrm{S} 2-\mathrm{H} 4, \mathrm{~S} 3-\mathrm{H} 5, \mathrm{~S} 4-\mathrm{H} 6$, and $\mathrm{H} 7-\mathrm{H} 8$ loops in NTD, providing the recognition specificity of eIF4A to PDCD4. At the center of the interface, Glu-210, Glu-249, and Asp-253 in mMA3 form an ion pair network with Arg-110 and Arg-161 from eIF4A (Fig. $2 A)$. The importance of this ion pair network is underscored by the previous observations that a mutation of Glu-249 or Asp-253 of PDCD4 significantly decreased the eIF4A-binding activity of PDCD4 and the D253A mutant failed to inhibit translation in vivo efficiently (10). In addition, in agreement with this struc- 
A

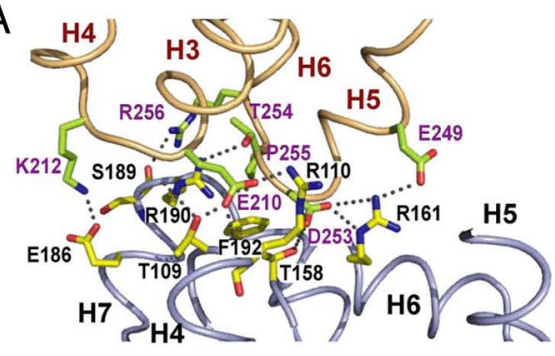

B

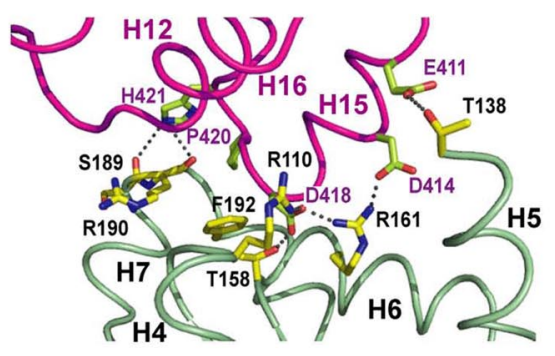

C

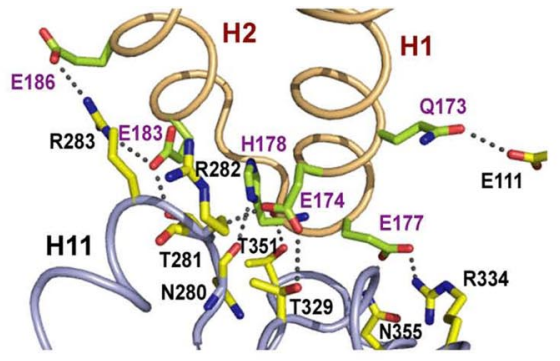

D

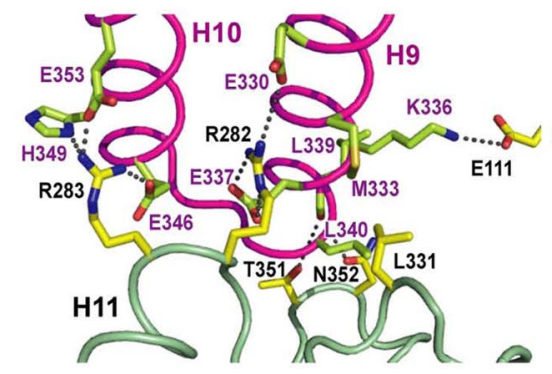

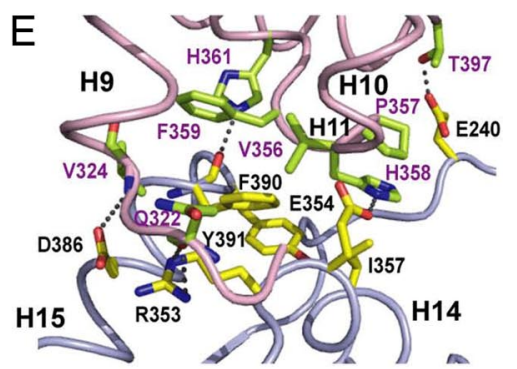

$\mathrm{F}$

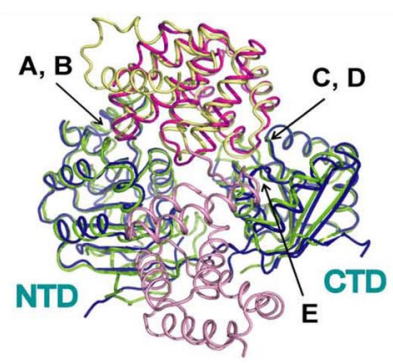

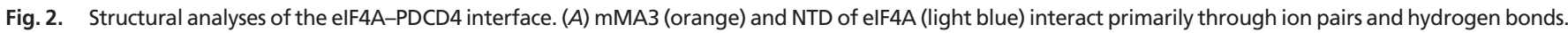

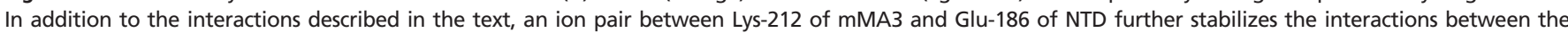

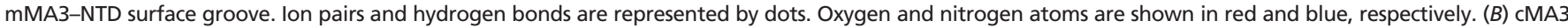

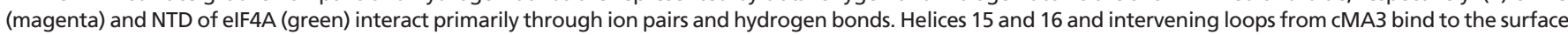

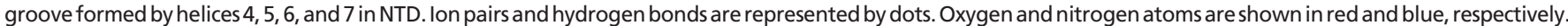

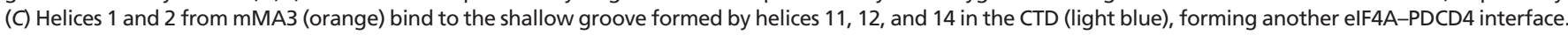
(D) Helices 9 and 10 from cMA3 (magenta) bind to the shallow groove formed by helices 11 and 14 in CTD (green), forming another interface. In addition to the

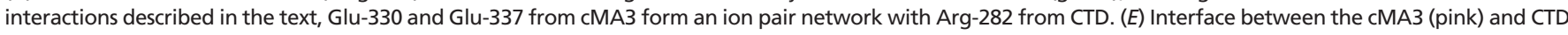
of elF4A (green). In addition to a hydrophobic cluster at the center of the interface, five hydrogen bonds and two ion pairs further stabilize this interface. ( $F$ ) Overall structure of the elF4A-PDCD4 complex in the same view of $A-E$.

tural feature, NMR studies revealed that Glu-210, Glu-249, and Asp-253 undergo chemical shifts upon binding to NTD of eIF4A (9). At the periphery of the network, Glu-210 of mMA3 hydrogen bonds with Thr-109 and Arg-110 of NTD, and Thr-254 and the backbone of Asp-253 in PDCD4 hydrogen bonds with Arg-190 in eIF4A, which buttress the central ion pair interaction.

At the mMA3-CTD interface, the $\mathrm{H} 1$ and $\mathrm{H} 2$ helices and an intervening loop of mMA3 recognize the surface groove formed by the S10-H11, S12-H12, and S13-H14 loops in the CTD. Specifically, Glu-183 and Glu-186 of mMA3 form a network with Thr-281 and Arg-283 of the CTD (Fig. 2C). In addition, Glu-111 and Asn-280 in eIF4A hydrogen bond with Gln-173 and His-178 in mMA3, respectively. In addition, Arg-334 and Asn-355 from CTD donate charge-stabilized hydrogen bonds to Glu-177 in mMA3.

Additional recognition specificities are provided by the interactions between cMA3 of PDCD4 and the CTD of eIF4A. Here, the interdomain linker of PDCD4 binds to the H15 helix and the S13-H14 loop of eIF4A (Fig. 2E), whereas the H11 helix and following loop of cMA3 bind to the surface groove formed by the H15-S16 and S13-H14 loops of the CTD. The hydrophobic cluster formed between Val-356, Pro-357, His-358, and Phe-359 in PDCD4 and Ile-357, Phe-390, and Tyr-391 in CTD appears to play an important role in the formation of the eIF4A-PDCD4 complex (see below).

cMA3 Domain of PDCD4 Is Sufficient to Interact with elF4A. In the cMA3-binding mode, the cMA3 domain recognizes a binding site in eIF4A. This binding site in eIF4A is shared with the mMA3 domain of PDCD4 in the two-MA3-binding mode (Figs. $1 B$ and $C$ and $2 A-D$ ). The mMA3 domain of PDCD4 in this mode is directed away from the interface of the two domains in eIF4A, toward the surface of CTD. The recognition interface of cMA3 is similar to that of mMA3 in the two-MA3-binding mode: the $\mathrm{H} 9$ and $\mathrm{H} 10$ helices bind to the surface groove of CTD, and the H15 and H16 helices and the following loop bind to the groove formed by the $\mathrm{H} 4$ and $\mathrm{H} 6$ helices and the $\mathrm{S} 3-\mathrm{H} 5$ and H7-H8 loops of NTD of eIF4A (Fig. $2 B$ and $D$ ). The extensive interface involving 2,255 $\AA$ of buried surface area with nine ion pairs and nine hydrogen bonds provides exquisite recognition specificity between cMA3 of PDCD4 and eIF4A in the cMA3binding mode. Asp-414 and Asp-418 form a central ion pair network with Arg-110 and Arg-161 of eIF4A; this network is similar to that observed between mMA3 and eIF4A in the two-MA3-binding mode. Previous mutational analysis and NMR titration studies revealed that Asp-414 and Asp-418 are involved in the binding to eIF4A, supporting the present structural observation $(1,9,10,12)$. Glu-346 and Glu-353 of cMA3 form an additional ion pair network with Arg-283 of eIF4A and stabilize the formation of the eIF4A-PDCD4 complex. In addition, Met-333 and Leu-340 of cMA3 make van der Waals contacts to Leu-331 of the CTD, thus contributing to the recognition of eIF4A by PDCD4 (Fig. 2D).

Mutational Analysis on the elF4A-PDCD4 Interface. To examine the importance of the interactions between eIF4A and PDCD4 observed in the cMA3-binding mode, we generated a series of single or double mutant proteins by replacing the eIF4Ainteracting residues on PDCD4 with alanine, and then we analyzed whether the mutations affected the formation of the eIF4A and PDCD 4 complex by using size exclusion chromatography (SEC) and ITC analyses. We selected the residues, Glu337, Met-333-Leu-340 (double mutant), Glu-346-Glu-353, and Asp-414-Asp-418 of PDCD4, which participate in the binding to eIF4A through the cMA3-binding mode (class 1 mutant). Based on circular dichroism analysis, it is unlikely that the mutations alter the overall structure of PDCD4 (Fig. S6). Both the N values from the ITC analysis and the shifted position of eluted peak in SEC analysis reveal that eIF4A and these PDCD4 mutant 

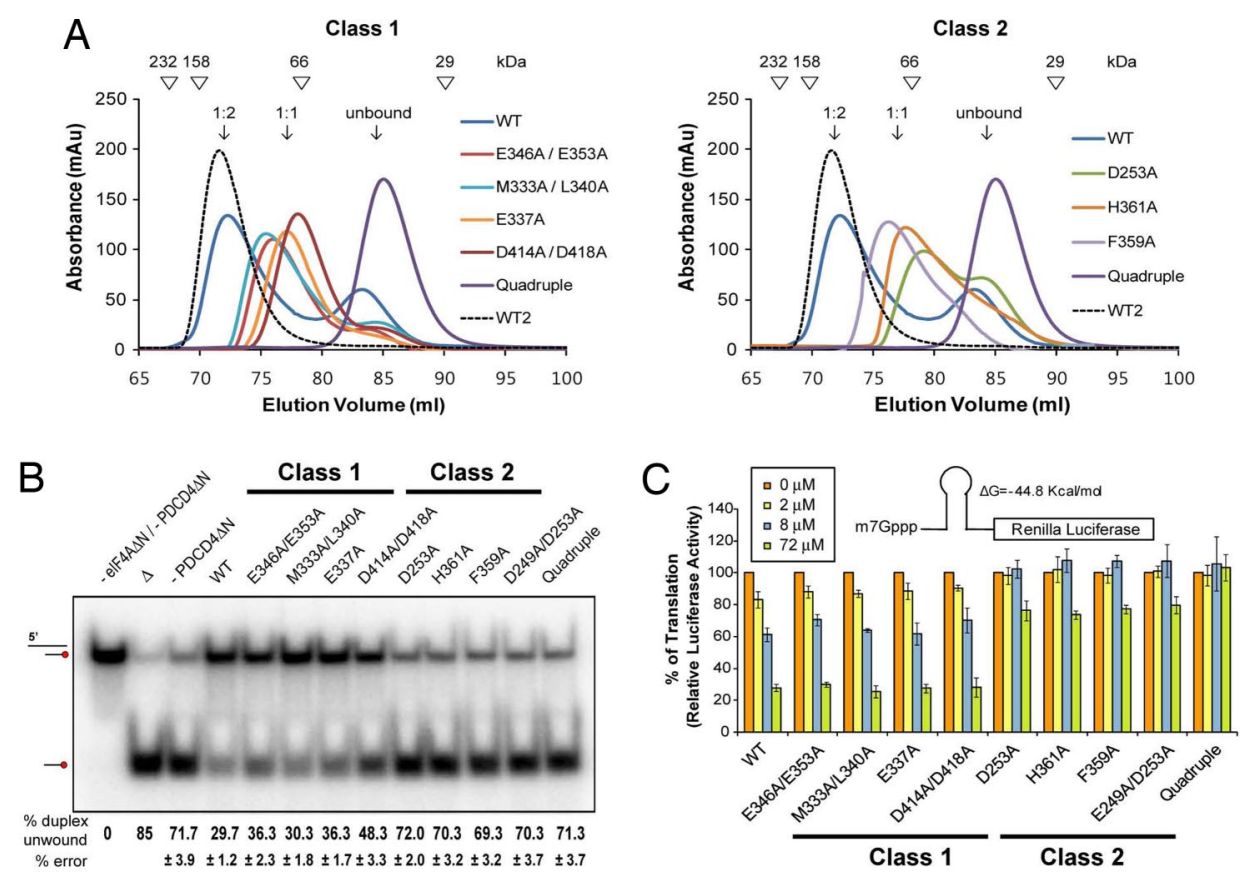

Fig. 3. Inhibitory activities of PDCD4 mutant proteins. ( $A$ ) (Left) SEC analysis of the elF4A $\triangle N-P D C D 4 \triangle N$ (class 1 ) mutant proteins. The quadruple mutant contains four mutated residues, E249A, E253A, D414A, and D418A. Before SEC analysis, the WT (blue line, $50 \mu \mathrm{M}$ ) or mutant PDCD4 $\triangle \mathrm{N}$ proteins and elF4A $\triangle \mathrm{N}$ were incubated at $4{ }^{\circ} \mathrm{C}$ for $1 \mathrm{~h}$ in a 1:1 molar ratio. WT2 (dotted line) represents the 2:1 elF4A $\triangle \mathrm{N}-\mathrm{WTPDCD} 4 \Delta \mathrm{N}$ complex. Although the WT2 complex shows no unbound elF4A or PDCD4 $\triangle N$, the WT complex exhibits notable amount unbound PDCD4. However, a 1:1 mixture of elF4A $\triangle N-P D C D 4 \triangle N$ mutant proteins did not show much of the free PDCD4 $\triangle N$. The standard molecular masses were the same as those in Fig. S1D. (Right) SEC analysis of the elF4A $\triangle N-P D C D 4 \Delta N$ (class 2) mutant proteins. $(B)$ Inhibition of elF4A helicase activities by various PDCD4 $\triangle N$ mutant proteins. The unwinding effects of 13-bp dsRNA by elF4A in the absence and presence of various PDCD $4 \triangle \mathrm{N}$ mutant proteins are shown. Total amount of unwound product was quantified by a Phosphorlmager and expressed as a percentage of total radiolabel (Bottom). $-4 A \Delta N /-P D C D 4 \Delta N$ and $\Delta$ represent reaction mixtures in the absence of elF4A at $0{ }^{\circ} \mathrm{C}$ and $95^{\circ} \mathrm{C}$, respectively. Each reaction mixture contains $2 \mathrm{nM}$ dsRNA, $3 \mu \mathrm{M}$ full elF4A, and $6 \mu \mathrm{M}$ various PDCD4 $4 \mathrm{~N}$ mutant proteins. Inhibition of elF4A by an excess amount of class 2 PDCD4 mutant proteins is shown in Fig. S7A. (C) In vitro translation inhibition by various PDCD4 $\triangle N$ mutant proteins. The percentage of translation occurring in the absence and presence of each mutant is indicated. The luciferase activity in the absence of WT or mutant PDCD4 $\Delta \mathrm{N}$ proteins was designated as $100 \%$. Different concentrations of various PDCD $4 \Delta \mathrm{N}$ mutants were used: orange bars, no PDCD $4 \Delta \mathrm{N}$; yellow bars, $2 \mu \mathrm{M}$; blue bars, $8 \mu \mathrm{M}$; green bars, $72 \mu \mathrm{M}$. Results indicate the means \pm SEM from triplicate experiments.

proteins form a 1:1 complex instead of the 2:1 complex, which suggests that the mutated residues are important for the formation of the eIF4A-PDCD4 complex (Fig. $3 A$, Fig. S2, and Table $\mathrm{S} 1$ ). The 2:1 mixture of the eIF4A-wild type (WT) PDCD4 complex forms a tight complex, and no free eIF4A or PDCD4 protein was eluted in SEC analysis, whereas a notable amount of unbound PDCD4 was eluted in the 1:1 mixture of the eIF4A-WT PDCD4 complex, supporting the 2:1 complex formation between eIF4A and WT PDCD4. However, only small amount of the unbound mutant PDCD4 proteins was eluted from the 1:1 mixture of the eIF4A-mutant PDCD4 complex (Fig. $3 A$ ).

We also examined the importance of ion pairs and van der Waals interactions in the formation of the eIF4A-PDCD4 complex in the two-MA3-binding mode (class 2 mutant). We replaced Asp-253 and Glu-249-Asp-253 from mMA3, and Phe359 and His-361 from cMA3 of PDCD4 with alanine. Gel filtration and ITC analyses revealed that the mutations on these residues altered the stoichiometry between the eIF4A and PDCD4 complex to 1:1 and decreased the affinities between the two proteins, which suggests that these PDCD4 residues are critical for the recognition of eIF4A (Fig. 3A, Fig. S2, and Table $\mathrm{S} 1)$. Interestingly, we observed a more dramatic effect on Asp-253 mutant compared with other single mutant, which suggests that Asp-253 plays a crucial role in the eIF4A-PDCD4 complex formation.

We then simultaneously mutated four residues of PDCD4 from the interface of the two different binding modes: judging from the gel filtration analysis, the mutation of Glu-249, Asp253, Asp-414, and Asp-418 to alanine abrogates the binding activity of PDCD4 toward eIF4A (Fig. 3A). Together, the mutational analyses support the present structural observation that the eIF4A and PDCD4 form a complex through both the two-MA3and cMA3-binding modes, and the interactions are achieved through several key ion pairs and hydrophobic interactions.

Implication of the Two Different Binding Modes in the Function of PDCD4. Although PDCD4 binds to eIF4A through two different binding modes, we were curious whether both binding modes are necessary for PDCD4 to inhibit the activity of eIF4A. To answer this question, we examined the inhibitory effects of the class 1 and 2 mutant PDCD4 proteins on the helicase activity of eIF4A and translation. As we have shown above, a mutant PDCD4 protein from each class forms a complex with eIF4A only through a single binding mode. First, we tested the inhibitory activities of the class 1 PDCD4 mutant proteins. In this class mutant (Glu-337, Met-333-Leu-340, Glu-346-Glu-353, and Asp-414-Asp-418), we perturbed the cMA3-binding mode in the eIF4A-PDCD4 complex, and thus, these mutant proteins could associate with eIF4A only through the two-MA3-binding mode (Fig. $2 B$ and $D$ and $3 A$ ). Fig. $3 B$ and $C$ shows that the mutant PDCD4 proteins of this class exhibit similar inhibitory effects on both the helicase activity of eIF4A and in vitro translation compared with those of WT PDCD4 (Fig. $3 B$ and $C$ ). These results suggest that the recognition of eIF4A by PDCD4 through the two-MA3-binding mode is important for the PDCD4mediated inhibition of the eIF4A and translation.

Previously, two mutational studies are reported on residues Asp-414 and Asp-418 of PDCD4 $(10,12)$. In one experiment, 
LaRonde-LeBlanc et al. (12) showed that the D414K and D418A mutant proteins of the isolated cMA3 fragment of PDCD4 failed to inhibit translation by using an in vivo transient expression system (12). These results are in agreement with our structural and mutational analysis because either Asp-414 or Asp-418 mutation of the cMA3 fragment would perturb the cMA3binding mode between the eIF4A and isolated cMA3 fragment of PDCD4 and dissociate the eIF4A-cMA3 complex. Interestingly, in another experiment, Yang et al. (10) showed that the mutation of Asp-414 to Ala in full-length PDCD4 also failed to inhibit the translation by using an in vivo transient expression system. This study is in contrast to our structure and mutational analysis. As we demonstrated above, the mutation of Asp-414 of PDCD4 is unlikely to affect the two-MA3-binding mode of PDCD4 on eIF4A binding. At present, it is unclear why the D414A mutant of full-length PDCD4 failed to inhibit the translation in vivo (10). Whereas we performed the PDCD4mediated inhibition assay by using in vitro translation analysis, Yang et al. examined the in vivo inhibition effect of PDCD4 by using transiently expression vector. Thus, there is a difference in the methods and the amount of proteins used in the two inhibition assays. Another possibility is that there might be some cooperative effect on PDCD4 and eIF4A binding, in vivo. If this is the case, only through both the two-MA3- and cMA3-binding modes, PDCD 4 effectively inhibits eIF4A, in vivo. Consequently, disruption of one binding mode of PDCD4 might fail to inhibit translation in vivo. However, as shown in our inhibition analysis, the cooperative binding of eIF4A on PDCD4 is unclear in vitro because only one mode of binding (two-MA3-binding) can efficiently inhibit eIF4A and translation (Fig. $3 B$ and $C$ ).

In contrast to the class 1 PDCD4 mutant proteins that interact with eIF4A through the two-MA3-binding mode, class 2 PDCD4 mutant proteins (Asp-253, Glu-249-Asp-253, Phe-359, and His$361)$ interact with PDCD4 only through the cMA3-binding mode (Figs. $2 A, C$, and $E$ and $3 A$ ). Interestingly, these mutant proteins exhibit some inhibitory effects on the helicase activity of eIF4A and in vitro translation only in the presence of large amounts of mutant proteins (Figs. $3 A-C$ and S7). Consistent with these data, a large amount of the mMA3 or cMA3 fragment of PDCD4 was required to inhibit eIF4A and the in vitro translation (Fig. S7). These results are also in agreement with a previous observation, in which the D253A mutant protein exhibits relatively weaker binding to eIF4A and reduced inhibition of in vivo translation compared with the D418A mutant protein (10). The differences in the inhibitory effect of PDCD4 between the two-MA3- and the cMA3-binding modes are primarily caused by the differences between the binding affinity of the two MA3 domains and the cMA3 alone toward eIF4A. We expect that both the two MA3 domains of PDCD4 would act synergistically to form a tighter complex with eIF4A compared with the cMA3 of PDCD4eIF4A complex. Supporting this idea, the calorimetry analysis revealed that PDCD $4 \Delta \mathrm{N}$ binds to eIF $4 A \Delta N>70$ times tighter than the cMA3 domain alone (Table S1). The quadruple PDCD4 mutant protein that does not interact with eIF4A lost its inhibitory effects on both helicase activity and in vitro translation (Fig. 3). Thus, our results demonstrate that PDCD4 inhibits the activities of eIF4A through each binding mode, although the two-MA3-binding mode is more effective for the inhibition of RNA helicase and translation compared with the cMA3-binding mode that weakly inhibits translation in vitro. Furthermore, the mutational analyses revealed a strong correlation between the reduced RNA helicase activity of eIF4A by PDCD4 and the PDCD4-mediated inhibition of translation, thereby supporting the idea that PDCD4 controls translation through the inhibition of eIF4A (1). Although the eIF4A and PDCD4 concentrations used in crystallographic and biochemical studies including size exclusion analysis are higher than those present in vivo, and this might not completely rule out some possibilities of
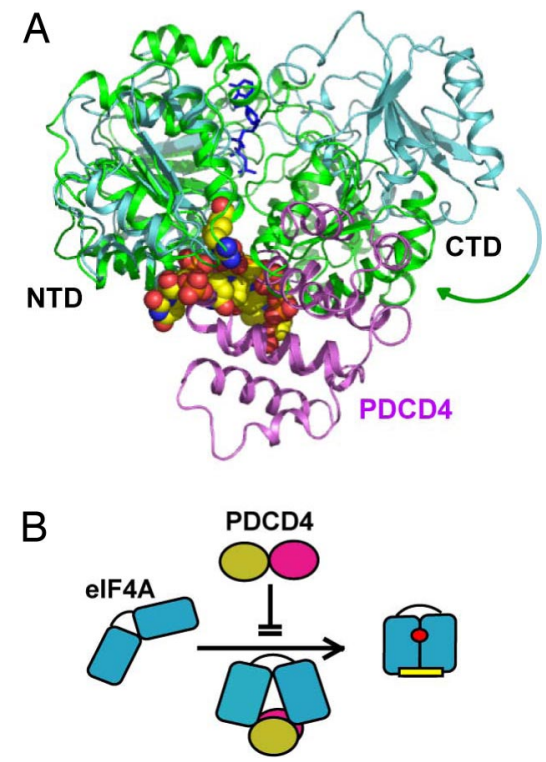

Fig. 4. Mechanism of the elF4A inhibition by PDCD4. (A) Structural superposition of the closed form of Vasa (green) that bound to dsRNA (space-filling model, oxygen in red, nitrogen in blue, and carbon in yellow) and the relatively open form of elF4A (light blue) that bound to CMA3 of PDCD4 (magenta) in the one-MA3 inhibition model. The CMA3 domain masks the dsRNA-binding site in elF4A. The ATP analog is indicated by a thick line (dark blue). Conformational change of PDCD4-bound elF4A to the closed form of Vasa is shown with an arrow. $(B)$ Schematic diagram of the PDCD4-mediated inhibition of elF4A. The domain closure movement of elF4A (blue) induced by ATP (small red circle) to unwind dsRNA (yellow) is blocked by the binding of the two MA3 domains (orange and magenta) of PDCD4 to the interface of elF4A.

nonspecific interactions between PDCD4 and eIF4A, the crystal structure clearly explains the molecular interactions between eIF4A and PDCD4 that elucidated from the our and previous mutational studies. In particular, the involvement of important residues, Asp-414, Asp-418, Glu-249, and Asp-253 of PDCD4 in eIF4A-PDCD4 complex formation can be only explained from the 2:1 eIF4A-PDCD4 complex that is confirmed in the present crystal structure and biochemical analysis.

Structural Basis for the Inhibition of elF4A by PDCD4. Previous NMR studies revealed that PDCD4 prevents eIF4A from binding to RNA (9). Comparison of the structure of the eIF4A-PDCD4 complex with that of an ATP analog- and RNA-bound Vasa, a Drosophila DEAD-box RNA helicase, revealed that PDCD4 masks a number of residues of human eIF4A that are involved in the recognition of RNA in eIF4A (16) (Fig. 4A). These residues include Arg-110, Thr-158, Arg-161, Glu-186, Asn-280, and Arg-282 of eIF4A (Fig. 3D). In particular, PDCD4 binds to the H6 helix (helix H7 in Vasa) in eIF4A. In Vasa, this helix plays a critical role in unwinding the base pairs by bending the RNA in the presence of ATP (16). Thus, PDCD4 blocks these conserved residues of eIF4A and impairs substrate recognition of eIF4A, supporting NMR observations for the inhibition of the RNA-binding activity of eIF4A by PDCD4.

The relative orientation of the two domains in the PDCD4bound eIF4A structure significantly differs from an "open form" of yeast eIF4A and a "closed form" of an ATP- and RNA-bound Vasa, which suggests that the flexible hinge joining the two RecA-like domains of eIF4A could allow a dynamic arrangement of the two domains (refs. 14 and 16 and Fig. S5). As proposed for the dsRNA melting mechanism of Vasa and other biochemical studies on eIF4A, we expect that eIF4A cooperatively binds to ATP and dsRNA in concert with the closure of the two 
domains and subsequently induces the unwinding of the bound dsRNA $(16,17)$ (Fig. 4A). In both of the two different modes of binding, PDCD 4 binds to the surface grooves of both domains of eIF4A and blocks the hinge-bending movement between the NTD and CTD of eIF4A (Fig. 4). This inhibition mechanism could be important because although PDCD4 may competitively inhibit RNA binding to eIF4A, it is still possible that RNA weakly associated with eIF4A in the presence of PDCD4. To inhibit eIF4A efficiently, multiple modes of inhibition mechanisms may be required for PDCD4. Our structure in conjunction with previous studies proposes that PDCD4 tightly inhibits eIF4A and translation initiation by $(i)$ displacing eIF4Gc and RNA from eIF4A $(1,14)$; (ii) masking the RNA-binding surface of eIF4A; and (iii) locking the domain closure of eIF4A.

The two marine natural products, pateamine A and hippuristanol, which regulate the activities of eIF4A, have recently been identified $(18,19)$. Although the detailed binding sites of these chemicals are not characterized, hippuristanol is known to bind to the CTD of eIF4A and impairs the RNA-binding activity of eIF4A, whereas pateamine A inhibits eIF4A-eIF4G association $(18,19)$. Our structure reveals that PDCD4 binds and regulates eIF4A differently from these compounds; PDCD4 recognizes both sides of the interdomain cleft of eIF4A (Fig. $1 E)$. Importantly, PDCD4 in the two different binding modes targets the same binding site within eIF4A, which suggests that the conserved surface grooves on both domains of eIF4A could be important binding sites for other MA3-containing proteins and/or for chemicals that mimic PDCD4. The structure of the eIF4A-PDCD4 complex suggests that blocking the domain closure could be another important strategy to inhibit the function of eIF4A.

Why would PDCD4 inhibit eIF4A through two different binding modes? In the flow of genetic information, regulation of gene expression at the level of translation is the last stage of expression regulation, and the response to environmental changes has to be rapidly and tightly controlled. Although we do not completely exclude the possibility that the two eIF4A molecules bind cooperatively to one PDCD4 molecule, in vivo, we propose that the two different binding modes might allow PDCD4 to overcome the mutational effects on a local region in a single MA3, which could otherwise abrogate the function PDCD4. In the 2:1 eIF4A-PDCD4 complex, multiple simulta-

1. Yang HS, et al. (2003) The transformation suppressor Pdcd4 is a novel eukaryotic translation initiation factor $4 \mathrm{~A}$ binding protein that inhibits translation. Mol Cell Biol 23:26-37.

2. Cmarik JL, et al. (1999) Differentially expressed protein PDCD4 inhibits tumor promoter-induced neoplastic transformation. Proc Natl Acad Sci USA 96:14037-14042

3. Jansen AP, Camalier CE, Colburn NH (2005) Epidermal expression of the translation inhibitor programmed cell death 4 suppresses tumorigenesis. Cancer Res 65:60346041.

4. Chen Y, et al. (2003) Loss of PDCD4 expression in human lung cancer correlates with tumour progression and prognosis. J Pathol 200:640-646.

5. Yang HS, Knies J, Stark C, Colburn NH (2003) PDCD4 suppresses tumor phenotype in JB6 cells by inhibiting AP-1 transactivation. Oncogene 22:3712-3720.

6. Jansen A, Camalier C, Stark C, Colburn NH (2004) Characterization of programmed cell death 4 in multiple human cancers reveals a novel enhancer of drug sensitivity. Mol Cancer Ther 3:103-110.

7. Dorrello NV, et al. (2006) S6K1- and $\beta$ TRCP-mediated degradation of PDCD4 promotes protein translation and cell growth. Science 314:467-471.

8. Gingras AC, Raught B, Sonenberg N (1999) elF4 initiation factors: Effectors of mRNA recruitment to ribosomes and regulators of translation. Annu Rev Biochem 68:913-963.

9. Suzuki C, et al. (2008) PDCD4 inhibits translation initiation by binding to elF4A using both its MA3 domains. Proc Natl Acad Sci USA 105:3274-3279.

10. Yang HS, et al. (2004) A novel function of the MA-3 domains in transformation and translation suppressor Pdcd4 is essential for its binding to eukaryotic translation initiation factor 4A. Mol Cell Biol 24:3894-3906.

11. Shibahara K, et al. (1995) Isolation of a novel mouse gene MA-3 that is induced upon programmed cell death. Gene 166:297-301. neous mutations on the two MA3 domains should be required to abrogate the function of PDCD4, and thus, interaction of PDCD4 with eIF4A through the two different binding modes provides an efficient way to prevent the aberrant protein synthesis in response to cellular stress.

In summary, our structural and functional studies on the eIF4A-PDCD4 complex suggest that PDCD4 efficiently and tightly inhibits eIF4A by multiple inhibition mechanism. The two different binding modes between eIF4A and PDCD4 explain the previous observations in which two MA3 domains of PDCD4 are involved in the interaction with eIF4A $(9,10)$, whereas cMA3 alone is sufficient to inhibit translation (12). The complex structure should serve as a framework to design therapeutics that mimic the PDCD4.

\section{Methods}

The human PDCD4 (residues 164-469) and elF4A (residues 20-406) were expressed and purified as a His-tagged form in Escherichia coli. Only elF4A contained $\mathrm{a} \mathrm{His} 6$ tag at its $\mathrm{N}$ terminus. To facilitate structure determination, we prepared the elF4A-PDCD4 complex by using Se-Met-substituted PDCD4. Further purification of the elF4A-PDCD4 complex was completed by using an anion exchange column (Mono Q) and a gel filtration column (Superdex 200). The protein complex was concentrated to $20 \mathrm{mg} / \mathrm{mL}$ by ultrafiltration. Crystals of the elF4A-PDCD4 complex were grown at $4{ }^{\circ} \mathrm{C}$ by using the hanging-drop vapor diffusion method. The well buffer contained $100 \mathrm{mM}$ sodium citrate $(\mathrm{pH}$ 6.0), 12-15\% 2-methyl 2,4-pentenediol (MPD), $100 \mathrm{mM}$ ammonium acetate, and 5 mM DTT. Diffraction data were collected with a crystal frozen at $-170^{\circ} \mathrm{C}$ at peak absorption, $0.9792 \AA$, on beamline 19ID at the Argonne National Laboratory. The crystal was prepared for freezing by equilibrating it in a buffer containing $30 \%$ ethylene glycol, $100 \mathrm{mM}$ sodium citrate $(\mathrm{pH} \mathrm{6.0)}$, $12-15 \%$ MPD, and $100 \mathrm{mM}$ ammonium acetate. The crystals belong to the spacegroup $\mathrm{P} 2{ }_{1} 2,2$ and contain two complexes per asymmetric unit. The unit cell has dimensions of $a=156.9 \AA, b=165.4 \AA$, and $c=100.4 \AA$. The structure of the elF4A-PDCD4 complex was determined by the single-wavelength anomalous dispersion method by using crystals containing Se-Met-derivatized PDCD4. Phase determination was carried out with SOLVE (20). After the flattening of solvent, a high-quality electron density map with a resolution of $2.8 \AA$ was obtained. The electron density was interpreted and traced by using Coot (21) and was refined with REFMAC using all reflections (22). Tight noncrystallographic symmetry restraints between the two complexes were applied throughout the refinement. Crystallographic statistics are summarized in Table S2. Full procedures are described in SI Methods.

ACKNOWLEDGMENTS. We thank WooJae Kim and JunHyun Kim for help with translation assays. This work was supported by the National Creative Research Initiatives.

12. LaRonde-LeBlanc N, Santhanam AN, Baker AR, Wlodawer A, Colburn NH (2007) Structural basis for inhibition of translation by the tumor suppressor Pdcd4. Mol Cell Biol 27:147-156.

13. Waters LC, et al. (2007) Structure of the C-terminal MA-3 domain of the tumour suppressor protein $\mathrm{Pdcd} 4$ and characterization of its interaction with elF4A. Oncogene 26:4941-4950.

14. Caruthers JM, Johnson ER, McKay DB (2000) Crystal structure of yeast initiation factor 4A, a DEAD-box RNA helicase. Proc Natl Acad Sci USA 97:13080-13085.

15. Schütz $P$, et al. (2008) Crystal structure of the yeast elF4A-elF4G complex: An RNA helicase controlled by protein-protein interactions. Proc Natl Acad Sci USA 105:95649569.

16. Sengoku T, Nureki O, Nakamura A, Kobayashi S, Yokoyama S (2006) Structural basis for RNA unwinding by the DEAD-box protein Drosophila Vasa. Cell 125:287-300.

17. Lorsch JR, Herschlag D (1998) The DEAD box protein elF4A. 2. A cycle of nucleotide and RNA-dependent conformational changes. Biochemistry 37:2194-2206.

18. Bordeleau ME, et al. (2006) Functional characterization of IRESes by a novel inhibitor of the RNA helicase elF4A. Nat Chem Biol 2:213-220.

19. Low WK, et al. (2005) Inhibition of eukaryotic translation initiation by the marine natural product pateamine A. Mol Cell 20:709-722.

20. Terwilliger TC, Berendzen J (1999) Automated MAD and MIR structure solution. Acta Crystallogr D 55:849-861.

21. Emsley P, Cowtan K (2004) Coot: Model-building tools for molecular graphics. Acta Crystallogr D 60:2126-2132.

22. Murshudov GN, Vagin AA, Dodson EJ (1997) Refinement of macromolecular structures by the maximum-likelihood method. Acta Crystallogr D 53:240-255. 